\title{
High magnetic field studies of the vortex lattice structure in $\mathrm{YBa}_{2} \mathrm{Cu}_{3} \mathrm{O}_{7}$
}

\author{
A. S. Cameron, ${ }^{1, *}$ J. S. White, ${ }^{2}$ A. T. Holmes, ${ }^{1}$ E. Blackburn, ${ }^{1}$ E. M. Forgan, ${ }^{1}$ R. Riyat,,${ }^{1}$ T. Loew, ${ }^{3}$ \\ C. D. Dewhurst, ${ }^{4}$ and A. Erb ${ }^{5}$ \\ ${ }^{1}$ School of Physics and Astronomy, University of Birmingham, Edgbaston, Birmingham, B15 2TT, United Kingdom \\ ${ }^{2}$ Laboratory for Neutron Scattering and Imaging, Paul Scherrer Institut, CH 5232 Villigen, Switzerland \\ ${ }^{3}$ Max Planck Institut für Festkörperforschung, D-70569 Stuttgart, Germany \\ ${ }^{4}$ Institut Laue-Langevin, 6 rue Jules Horowitz, 38042 Grenoble, France \\ ${ }^{5}$ Walther Meissner Institut, BAdW, D-85748 Garching, Germany
}

(Received 12 March 2014; revised manuscript received 14 July 2014; published 4 August 2014)

\begin{abstract}
We report on small-angle neutron scattering (SANS) measurements of the vortex lattice (VL) in twin-free $\mathrm{YBa}_{2} \mathrm{Cu}_{3} \mathrm{O}_{7}$, extending the previously investigated maximum magnetic field of $<11 \mathrm{~T}$ up to $16.7 \mathrm{~T}$ with the field applied parallel to the $\mathbf{c}$ axis. This is a microscopic study of vortex matter in this region of the superconducting phase. We find that the high magnetic field VL displays a rhombic structure, with a field-dependent coordination that passes through a square configuration with no lock-in to a field-independent structure. The VL pinning reduces with increasing temperature but is seen to affect the VL correlation length even above the irreversibility temperature of the lattice structure. At high field and temperature we observe a VL melting transition, which appears to be first order. The vortex liquid phase above the transition does not give a detectable SANS signal.
\end{abstract}

DOI: 10.1103/PhysRevB.90.054502

PACS number(s): 74.25.Wx, 74.25.Ha, 74.72.Gh, 25.40.Dn

\section{INTRODUCTION}

$\mathrm{YBa}_{2} \mathrm{Cu}_{3} \mathrm{O}_{7-\delta}$ (YBCO) was the first high- $T_{c}$ superconductor in which the mixed state was studied by small-angle neutron scattering (SANS) [1]. In the more than 25 years since the discovery of YBCO, sample quality has greatly improved and the available magnetic field range has increased, allowing increasingly sophisticated studies of the mixed state by a variety of techniques [1-19]. In particular, SANS measurements have given direct microscopic information about vortex lines in the mixed state in bulk YBCO [1,8-19]. These measurements lead to an understanding of the physics of the vortex lattice (VL) and the effects of crystal anisotropy, thermal excitation, and pinning on the VL structure and its thermal melting. Furthermore, the observed properties of the VL can give information about the superconducting pairing state in this material and its evolution with field. Here we report on SANS studies of the VL in fully oxygenated $\mathrm{YBa}_{2} \mathrm{Cu}_{3} \mathrm{O}_{7}$ $\left(\mathrm{YBCO}_{7}\right)$. This material is slightly overdoped, and pinning on oxygen vacancies of magnetic vortex lines in the mixed state is greatly reduced by full oxygenation. We extend the field range of previous SANS studies from 10.8 to $16.7 \mathrm{~T}$, providing microscopic structural information about the behavior of the $\mathrm{VL}$ at those fields in a high- $T_{c}$ material.

YBCO has an orthorhombic crystal structure containing two-dimensional $\mathrm{CuO}_{2} a b$-plane layers, and one-dimensional $\mathrm{CuO}$ chain layers running along the $\mathbf{b}$ direction. These are stacked along the $\mathbf{c}$ direction, separated by $\mathrm{Y}$ and $\mathrm{BaO}$ layers. As in all high- $T_{c}$ materials, superconductivity with a nodal gap structure is mainly localized in the $\mathrm{CuO}_{2}$ layers. However, for optimally doped and overdoped compositions, the $\mathrm{CuO}$ chains display both long-range order along the crystal $\mathbf{b}$ axis and metallic behavior [20]. As a consequence, it is expected that the electronic states primarily associated with the chains are also superconducting below $T_{c}$. This picture

*a.cameron2478@googlemail.com is supported by reported values of the in-plane penetration depth ratio, $\gamma_{\lambda}\left(=\lambda_{a} / \lambda_{b} \propto \sqrt{m_{a}^{*} / m_{b}^{*}}\right)$, which lie in the range $1.2-1.6[7,15,18,19,21-23]$. The direction of this anisotropy is that expected if there is a contribution to the superfluid density along b from the chain carriers. The observed anisotropy cannot be due the plane carriers, because the anisotropy between the $\mathbf{a}$ and $\mathbf{b}$ hopping matrix elements for these carriers given by local-density approximation (LDA) band calculations [24] is close to unity and in the wrong direction. It has been suggested that the chain pairing may arise from a proximity-effect single-particle coupling between chain and plane states [25-27], but an alternative cause is a Josephson pair coupling [28,29]. It has been pointed out [30] that the superfluid density along $\mathbf{a}$ and $\mathbf{b}$ both show a $d$-wave temperature dependence at low $T[18,19]$. This is consistent with intrinsically superconducting chains coupled to the planes by a Josephson-type pair tunneling [28]. In this case, the $d$-wave behavior for the $\mathbf{b}$ direction implies that there are nodes in the superconducting gap for the chain carriers also. The positions of these nodes on the chain Fermi surface would not be fixed by symmetry and could move as a function of field and temperature. A further consequence of the crystal orthorhombicity is that the predominantly $d_{x^{2}-y^{2}}$ order parameter of the plane carriers must contain a finite additional $s$-wave component [31] This implies that the nodes on the plane Fermi surface are not exactly at $45^{\circ}$ to the crystal axes. Evidence for this admixture is provided by phase-sensitive [32], tunneling [33], and muon spin rotation ( $\mu \mathrm{SR})[34]$ studies.

As-grown YBCO crystals are crystallographically twinned; the twin planes running at approximately $45^{\circ}$ between adjacent $\mathbf{a}$ and $\mathbf{b}$ domains act as pinning centers. These have had a clear influence on the results from almost all previous SANS studies [1,8-17]. Detwinned samples have only been used in the most recent investigations $[18,19]$. These show first-order phase transitions between different VL phases in contrast to the continuous transitions reported in previous studies $[15,17]$. 
Our present samples are detwinned and allow us to follow the intrinsic behavior of the VL in YBCO into a new field range, where the vortex cores are closer together and the effect of the order parameter anisotropy on the VL structure is expected to be more pronounced. In addition to this, the intensity of the SANS signal as a function of field and temperature depends on the magnitude of the spatial variation of magnetic field in the mixed state. This is measured in terms of the VL "form factor," which reflects the coherence length $\xi$ of the Cooper pairs, the magnetic penetration depth $\lambda$, and the departure of the vortex lines from straightness, represented by a Debye-Waller factor. For our field range it is expected that the VL undergoes a melting transition to a vortex liquid on heating. SANS data can complement existing thermodynamic measurements [35] by adding a unique insight into the VL structure and perfection near the melting transition.

\section{EXPERIMENTAL}

The sample was a mosaic of six single crystals of detwinned $\mathrm{YBa}_{2} \mathrm{Cu}_{3} \mathrm{O}_{7}$ with a total mass of $\sim 20 \mathrm{mg}$. Each crystal was grown from a molten flux of $\mathrm{BaCO}_{3}, \mathrm{CuO}$, and $\mathrm{Y}_{2} \mathrm{O}_{3}$ in $\mathrm{BaZrO}_{3}$ crucibles [36]. Upon cooling from the hightemperature tetragonal phase to the orthorhombic phase, the inequality between the crystal $\mathbf{a}$ and $\mathbf{b}$ axes results in crystallographic twinning. It has been seen in previous SANS studies [15] that vortex lines are strongly pinned to the twin planes, dominating the VL orientation at the expense of other physical phenomena. To remove this effect, these crystals were detwinned through the application of uniaxial stress at $500{ }^{\circ} \mathrm{C}$ for $24 \mathrm{hr}[37,38]$. The crystals were then oxygenated under an $\mathrm{O}_{2}$ atmosphere of 100 bars at $300{ }^{\circ} \mathrm{C}$ for $150 \mathrm{hr}$ [39]. A crystal from the mosaic gave a zero field $T_{c}$ of $89.0 \mathrm{~K}$ by superconducting quantum interference device (SQUID) magnetometry in a field of $1 \mathrm{mT}$, with a $90 \%$ superconducting transition width of $2 \mathrm{~K}$. The mosaic was mounted on a 1-mmthick pure aluminum plate, with the crystal $\mathbf{c}$ axis perpendicular to the plate and the a direction co-aligned between crystals.

The experimental work was carried out on the D22 instrument at the Institut Laue-Langevin in Grenoble, France. The mosaic was mounted on a variable-temperature stage in a horizontal-field cryomagnet of novel design [40], with the crystal $\mathbf{c}$ axes parallel to the applied field and the a axes vertical. SANS measurements were performed with the neutron beam entering at a range of angles close to the field direction. Neutrons of mean wavelength of 6-10 $\AA$ were used with a $10 \%$ FWHM wavelength spread, collimated over a distance of $8 \mathrm{~m}$.

For all measurements, the vortex lattice was prepared using the oscillation field cooled (OFC) procedure, where the sample is cooled through $T_{c}$ in an applied field with a small $(\sim 0.1 \%)$ oscillation about the required field. This method was chosen because a previous investigation [18] showed that this allowed the VL to find its preferred structural free energy minimum for an effective temperature lower than for cooling in a static field. The diffraction patterns were acquired by rocking the sample and field together through the required Bragg conditions, producing what is known as a "rocking curve," shown in the inset of Fig. 2. Background measurements were taken above $T_{c}$ and subtracted from the in-field measurements to leave only the signal from the vortex lattice.
Data visualization and analysis were performed using the GRASP analysis package [41]. This allowed the determination of the fitted positions and angles of VL diffraction spots and the evaluation of rocking curve widths and intensities integrated over rocking curves. Within the mixed state, the local field is expressed as a sum over spatial Fourier components at the two-dimensional reciprocal lattice vectors $\mathbf{q}$ of the VL. The magnitude of a Fourier component $F(\mathbf{q})$ is known as the form factor at wave vector $\mathbf{q}$. Its value is obtained from the integrated intensity $I_{\mathbf{q}}$ of a VL reflection as the VL is rocked through the Bragg condition for a diffracted spot at scattering vector $\mathbf{q}$. The vortex lattice form factor $F(\mathbf{q})$ is related to the integrated intensity $I_{\mathbf{q}}$ via the relationship [42]

$$
I_{\mathbf{q}}=2 \pi V \phi\left(\frac{\gamma}{4}\right)^{2} \frac{\lambda_{n}^{2}}{\Phi_{0}^{2} q}|F(\mathbf{q})|^{2} .
$$

Here, $V$ is the illuminated sample volume, $\phi$ is the incident neutron flux, $\lambda_{n}$ is the neutron wavelength, $\gamma$ is the magnetic moment of the neutron in nuclear magnetons $(=1.91)$, and $\Phi_{0}=h / 2 e$ is the flux quantum. The integrated intensity was determined by fitting the rocking curves to a Lorentzian line shape. When appropriate, $I_{\mathbf{q}}$ was corrected by the cosine of the angle between the scan direction and $\mathbf{q}$ (the Lorentz factor) [43]. In the work presented here, we measured the form factor for the first-order reflections which are all equivalent, so we may write it as $F(\mathbf{q})$.

\section{RESULTS AND DISCUSSION}

\section{A. Vortex lattice structure}

Previous VL studies on $\mathrm{YBCO}_{7}[18,19]$, up to $10.8 \mathrm{~T}$, showed first-order transitions from low magnetic field hexagonal structures to a high field rhombic phase. At high fields, the VL structure evolved towards a square configuration. Figure 1(a) shows a typical (not quite square) diffraction pattern obtained at high field and base temperature. Figure 1(b) shows the evolution of the VL shape as a function of magnetic field from 8 to $16.7 \mathrm{~T}$ for two different temperatures, and panel (c) shows the evolution with temperature on warming from a base of $2 \mathrm{~K}$ for two different fields. The evolution with field is seen to continue, passing through a square configuration at around $12.5 \mathrm{~T}$ at base temperature.

London theory with nonlocality (NL) corrections has had some success in describing the VL of superconductors with a fourfold axis $[44,45]$ and in a conventional high- $\kappa$ material such as $\mathrm{V}_{3} \mathrm{Si}$ [46]. NL can give extra anisotropy about a crystal axis, in addition to the effective mass anisotropy in local theories. They can arise from two causes: either Fermi surface anisotropy, which was initially treated in combination with a constant superconducting energy gap $\Delta[44,45]$ and/or from an anisotropic $\Delta[44,47,48]$. In $d$-wave materials, the effective coherence length $\xi \propto 1 / \Delta(k)$, such that nonlocal effects must always be important near nodes in the energy gap. However, for fields up to $11 \mathrm{~T}$, none of the NL theories agree with the observed structural evolution of the VL in $\mathrm{YBCO}_{7}$, an observation which continues to hold for the present measurements [19]. We therefore prefer to rely on the predictions of microscopic calculations using the Eilenberger theory [49]. From these it is predicted that at high fields the 

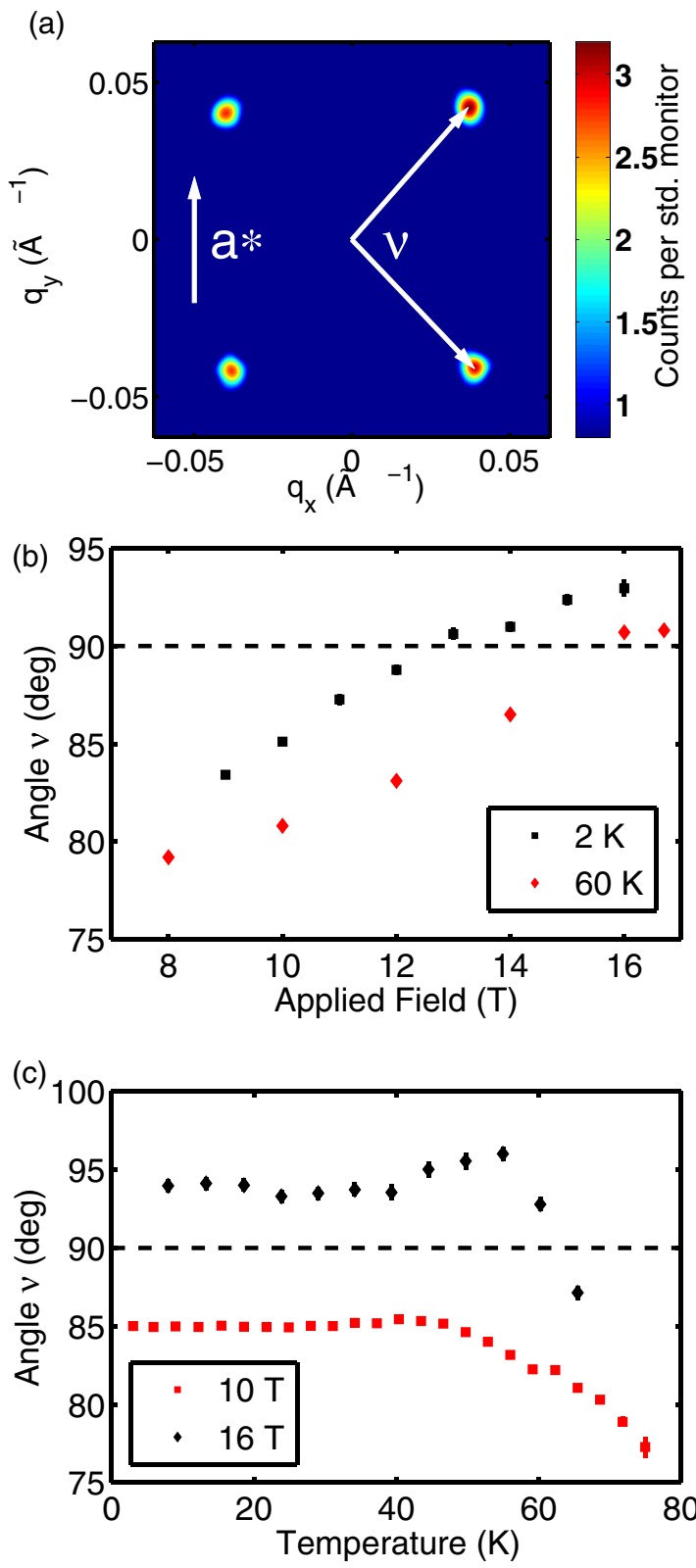

FIG. 1. (Color online) (a) Typical diffraction pattern from the $\mathrm{VL}$, taken in a field of $16 \mathrm{~T}$ at $2 \mathrm{~K}$. The structure of the VL may be described by the opening angle $v$. (b) Graph showing the structural evolution of the VL as a function of applied field. Measurements were taken after cooling while the field was oscillated slightly in value $(\mathrm{OFC})$ to $60 \mathrm{~K}$ or $2 \mathrm{~K}$, for fields in the range $8-16.7 \mathrm{~T}$. (c) Values of $v$ for a range of temperatures at applied fields of 10 and $16 \mathrm{~T}$, taken on warming from base temperature after OFC.

nearest-neighbor vortex directions will lie along the nodal directions of the order parameter. Our observations of the VL structural evolution suggest a field-driven change in the nodal positions, and we propose that superconducting states on the $\mathrm{CuO}$ chains may be responsible for this.

The role of the chains in the superconductivity of $\mathrm{YBCO}_{7}$ is illustrated by the value of the low field London penetration depth anisotropy $\sim 1.28[18,19]$, which implies an anisotropy in $n_{s} / m^{*}$ of $>1.6$. As emphasized earlier, this value is higher than can be accounted for by plane superconductivity alone and the sign of the anisotropy implies an extra contribution to superfluid density $n_{s}$ from the chains. Furthermore, it is clear from the temperature dependencies of $n_{s}$ that the superconducting order parameter has nodes on both the plane and chain Fermi surfaces [30]. However, the the quasi-one-dimensional chain superconductivity appears to be less robust than its plane counterpart, since the $a b$ anisotropy reduces with field $[18,19]$. The behavior of the chain states in $\mathrm{YBCO}_{7}$ is distinctly different from the chain states in $\mathrm{YBa}_{2} \mathrm{Cu}_{4} \mathrm{O}_{8}$ [30]. That compound requires a multigap description, which corresponds to a proximity-effect single-particle coupling between chain and plane states. This is in contrast to the single-gap behavior in $\mathrm{YBCO}_{7}$, which corresponds to a Josephson-like pair tunneling model for chain/plane coupling. This rules out a common description for chain-state behavior in Y-based cuprates. In the data presented here, we see that the anisotropy changes sign at high fields, since the VL structure passes through square coordination. Since we are at a small fraction of $H_{c 2}$ even at $17 \mathrm{~T}$, we propose that this is an indication of a modification of the weaker chain superconductivity with field; in particular, the nodal positions on the chain Fermi surface may move. New ARPES experiments to confirm chain nodes at zero field, and high field scanning tunnel microscopy (STM) or detailed calculations may be able to confirm these ideas.

Figure 1(c) shows the evolution of the VL structure with temperature. We first consider the behavior near $T_{c}$. It is predicted $[44,47,48]$ that NL will be reduced on approaching $T_{c}$. This suppression would bring the VL structure towards the hexagonal structure expected by local electrodynamics, which is in general agreement with the results in Fig. 1(c). The nonmonotonic variation with temperature seen near $40-60 \mathrm{~K}$ is surprising; we believe this is intrinsic but since it occurs at a temperature where pinning effects are changing, we postpone discussion until these are considered.

\section{B. Variation of form factor with field}

Now we turn to the spatial variation of magnetic field within the VL. To model the field dependence of the form factor, we have used the the local London model, extended to account for the $a b$ plane anisotropy of $\mathrm{YBCO}_{7}$, and including a Gaussian cutoff term to account for the finite size of the vortex cores [19]:

$$
F(\mathbf{q})=\frac{\langle B\rangle \exp \left[-c\left(\mathbf{q}_{x}^{2} \xi_{b}^{2}+\mathbf{q}_{y}^{2} \xi_{a}^{2}\right)\right]}{\mathbf{q}_{x}^{2} \lambda_{a}^{2}+\mathbf{q}_{y}^{2} \lambda_{b}^{2}} .
$$

Here, $\langle B\rangle$ is the average internal induction, $\lambda_{i}$ is the penetration depth arising from supercurrents flowing in direction $i, \xi_{i}$ is the coherence length along $i$, and $\mathbf{q}_{x}, \mathbf{q}_{y}$ are in-plane Cartesian components of the scattering vector, with $\mathbf{q}_{x}$ parallel to $\mathbf{b}^{*}$. The cutoff parameter $c$ accounts for the finite size of the vortex cores, and a suitable value for $c$ in our field and temperature range is 0.44 [19].

Using the values for $\xi$ and $\lambda$ from previous experiments [19], with $\lambda_{a}=138 \mathrm{~nm}, \lambda_{b}=107 \mathrm{~nm}, \xi_{a}=3.04 \mathrm{~nm}$, and $\xi_{b}=3.54 \mathrm{~nm}$, labeled as the "Low field" model in Fig. 2, we find that the form factor above $10 \mathrm{~T}$ is much larger than the extrapolation from low field data. Taking smaller values of $\xi_{a}=2.60 \mathrm{~nm}$ and $\xi_{b}=3.03 \mathrm{~nm}$ with $\lambda$ unchanged provides the "High field" line in Fig. 2; however, this departs from the lower field data. Indeed, we find that no physically reasonable 


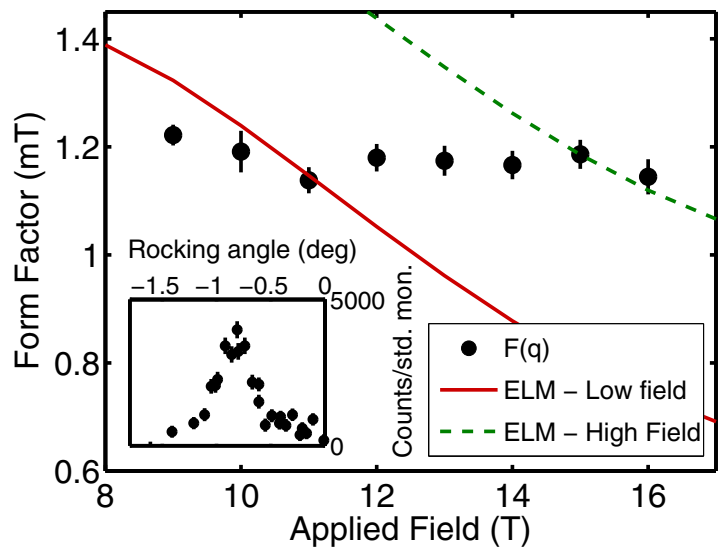

FIG. 2. (Color online) The variation with field of the VL form factor for the 1st-order diffraction spots [shown in Fig. 1(c)] at a temperature of $2 \mathrm{~K}$. Integrated intensities were obtained by fitting the data to a Lorentzian line shape. The solid red line shows the extended London model (ELM) prediction [Eq. (2)], using parameter values obtained by fitting earlier data taken below $11 \mathrm{~T}$ [19], while the dotted green line shows a prediction, discussed in the text, from the ELM using smaller values of $\xi$.

constant values of the parameters fit the full field range of our data.

As noted earlier [19], the low field fitted values for $\xi_{a}$ and $\xi_{b}$ are larger than expected and therefore not intrinsic, because the upper critical field estimated from them is too low for $\mathrm{YBCO}_{7}$. These authors suggested that instead they reflect pinning effects. These remarks remain true for the present high field results. The larger values of $\xi$ probably reflect frozen-in disorder in the VL giving a static Debye-Waller (DW) factor, reducing the scattered intensity. A mean square deviation $\left\langle u^{2}\right\rangle$ from straightness would contribute an additional term $\exp \left(-\mathbf{q}^{2}\left\langle u^{2}\right\rangle / 4\right)$ to Eq. (2), which has the effect of increasing the Gaussian cutoff term and simulating a larger vortex core size. We deduce that the effects of disorder in our high magnetic field range are relatively smaller, and that the actual values of $\xi$ are smaller than those obtained from fits.

We note that disorder in the VL leading to an overestimation of the coherence length $\xi$ from SANS has been observed in other high- $T_{c}$ materials. The superconductor $\mathrm{La}_{2-x} \mathrm{Sr}_{x} \mathrm{CuO}_{4}$ (LSCO) gave distinctly different values for $\xi$ from SANS in comparison to heat capacity measurements [50,51]. This was attributed to disorder in the VL leading to a similar overestimation of the coherence length to the situation we have here, although the disorder in LSCO was driven by electronic effects rather than the static pinning as is the case in close-to-optimally doped YBCO.

\section{Variation of the vortex lattice with temperature}

Figure 3 shows the variation of VL form factor and rocking curve width (FWHM) with temperature. The rocking curve width is the combined effect of both instrument resolution and the perfection of the VL [52], and is related to the correlation length $\xi_{L}$ of the vortices along the field direction [53]. It can be seen that VL disorder dominates away from $T_{c}$. From such data, we can identify an "irreversibility temperature" of $\sim 40$

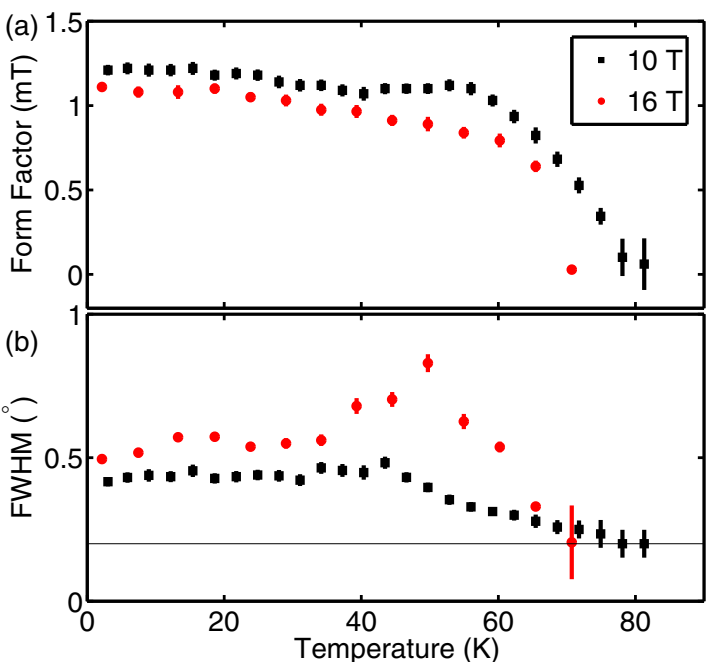

FIG. 3. (Color online) (a) The variation with temperature of the VL form factor at 10 and $16 \mathrm{~T}$, with data taken on warming after OFC. The integrated intensity was calculated by fitting the rocking curves to a Lorentzian line shape. (b) Rocking curve widths vs temperature, compared with the resolution width of the instrument (solid line).

$50 \mathrm{~K}$ below which the VL is "frozen in" and the rocking curve width and structure [Fig. 1(b)] do not change. Close to $T_{c}$, the FWHM of both data sets lie above the resolution limit, showing that pinning can still distort the vortex lines away from straightness even though the VL as a whole can move. Near the irreversibility temperature, the 10 and $16 \mathrm{~T}$ data exhibit differing behaviors, with the rocking curve width at $10 \mathrm{~T}$ decreasing towards $T_{c}$, while at $16 \mathrm{~T}$ the width increases before also narrowing on the approach to $T_{c}$. In the same region, the opening angle of the VL structure in Fig. 1 has a peak. Further measurements, not reported here, show that this occurs both on warming and cooling, indicating that this is not due to annealing of the VL on warming. We speculate that also in this case, temperature- and field-dependent changes in the nodes, particularly in the chain order parameter, may be influencing the VL structure and pinning.

The temperature dependence of $F(\mathbf{q})$ in Fig. 3, particularly at $10 \mathrm{~T}$, may also represent such intrinsic effects, although we cannot rule out the effects of changes in a static DW factor. However, well below the irreversibility temperature both the VL structure and its lattice perfection remain fixed; hence the temperature dependence of $F(\mathbf{q})$ should reflect intrinsic properties of the sample. It has been suggested that the weak temperature dependence of $F(\mathbf{q})$ seen at low temperatures is due to a stronger NL effects in large fields [19,54]. Following the nonlocal model employed earlier [19] to fit data from YBCO at lower fields, we find that using the parameters for $\xi$ and $\lambda$ from Fig. 2 provides a fit to the data at base temperature, and allowing these parameters to vary provides a good fit up to around $40 \mathrm{~K}$. Above this region we expect that the static DW factor would reduce as the VL becomes able to move. This reduction in disorder leading to an increase in SANS intensity from a vortex lattice was first observed in $\mathrm{Bi}_{2} \mathrm{Sr}_{2} \mathrm{CaCu}_{2} \mathrm{O}_{8+\delta}$ (BSCCO) [55], where thermal fluctuations at higher temperature was seen to smooth out the pinning 
potentials for the VL, leading to a re-emergence of the vortex lattice signal at higher temperatures. The effect of thermal fluctuations in YBCO, however, appears far less pronounced since the material is much more isotropic than BSCCO, and as such the vortex lines in YBCO do not decompose into vortex pancakes and the disorder is correspondingly much lower.

\section{Vortex lattice melting}

In Fig. 3, the form factor at $16 \mathrm{~T}$ shows a sudden drop close to $70 \mathrm{~K}$. A more detailed scan between 60 and $80 \mathrm{~K}$ at $16.7 \mathrm{~T}$ is shown in Fig. 4(a), together with a solid line giving the superfluid density from the 3D-XY model [56]. This model can be used to give the VL form factor as

$$
F \propto\left[1-T / T_{c 2}(B)\right]^{n},
$$

with $T_{c 2}(B)$ taken from work by Junod et al. [57], and $n=0.66$. It can be seen in Fig. 4(a) that the form factor data drop well below the model prediction. The falloff will begin as a (true) Debye-Waller effect arising from the increasing thermally induced excursions of the VL from equilibrium, followed by VL melting when the displacements become a large enough fraction $c_{L}$ of the the vortex line spacing [58]. Here, $c_{L}$ is called the Lindemann number and is expected to lie in the range $0.15-0.3$. We can estimate the reduction of intensity due to the DW factor, $\exp \left(-\mathbf{q}^{2}\left\langle u^{2}\right\rangle / 2\right)$, using

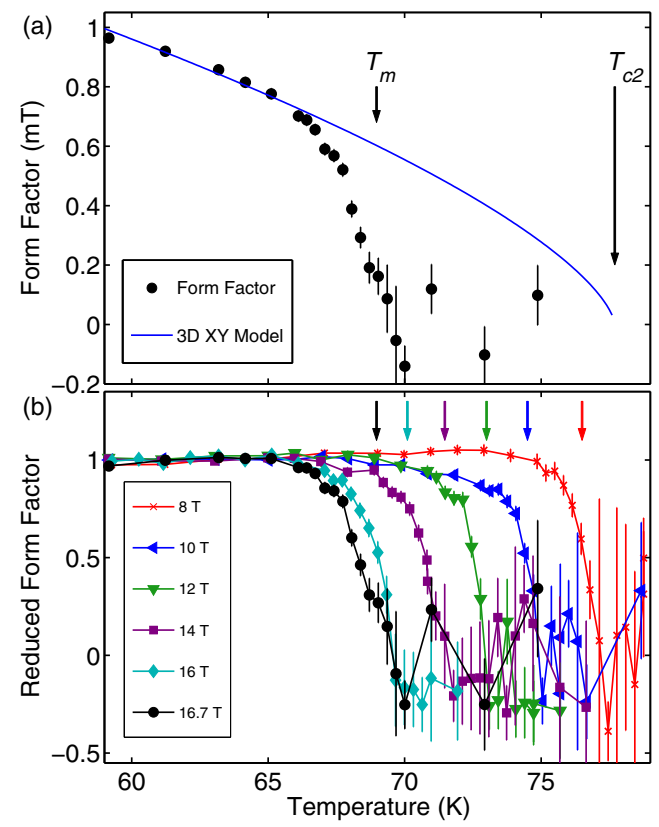

FIG. 4. (Color online) Variation with temperature of $F(\mathbf{q})$ across the VL melting transition. (a) The raw form factor for an applied field of $16.7 \mathrm{~T}$, alongside the predicted variation from the 3D-XY model. (b) The reduced form factor for fields between 8 and $16.7 \mathrm{~T}$, with the reduced form factor obtained by dividing the data by the prediction from the 3D-XY model. The arrows indicate melting temperatures, obtained from a linear fit vs temperature of the fall to zero of the intensity. The noise above the melting temperature appears larger because of the square root relating intensity to form factor, and also because the plotted quantity is divided by the function in Eq. (2), which is tending to zero. $\left\langle u^{2}\right\rangle=c_{L}^{2} a^{2}$, where $a$ is the spacing between vortex lines. With $c_{L}=0.2$, we find that before melting, the intensity of a first-order diffracted spot would already have fallen to a fraction $\sim 0.45$ of that for a perfect lattice. In Fig. 4(b) are shown the data for a range of fields plotted as a fraction of the 3D-XY variation. The melting line $B_{m}(T)$ derived from these results is very close to that found by heat capacity measurements [35] of the lattice-liquid transition. However, in addition, the present data give microscopic information about the VL structure as melting occurs. Macroscopic measurements have shown that a transition point between firstand second-order melting is dependent on pinning disorder and oxygen content $[34,35,59]$. First-order melting is expected in our case, because macroscopic measurements on $\mathrm{YBCO}_{7}$ from the same source as our sample show that the first-order transition continues up to $\sim 30 \mathrm{~T}[60]$. We hesitate to compare our SANS observations of VL melting in $\mathrm{YBCO}_{7}$ with other observations using the same technique, because all other measurements were on very different materials. The high- $T_{c}$ superconductor BiSCCO is a very anisotropic material, so that the vortex lines are very flexible and easily disrupted by pinning or thermal fluctuations; VL melting occurs at far lower fields than in YBCO, although there are some interesting suggestions of DW effects in the data $[55,61]$. An earlier paper on YBCO [13] reported results in a much lower field range on a heavily twinned sample, while observations on an ultra-pure sample of niobium using both SANS and high-resolution heat capacity techniques [62] surprisingly show no sign of analogous VL melting in this nonlayered low- $T_{c}$ material.

The intensity of Bragg reflections is predicted to drop sharply to near zero upon melting of the VL due to the loss of crystalline order, which would smear the spots into a ring, and we estimate this would reduce the signal at the reflection by an order of magnitude. However, even after counting for an extended period, no signal from the vortex liquid was detected above the transition. Should the rocking curve width of the vortex liquid remain similar to that of the vortex solid (and we note that the FWHM of the VL remained narrow during the melting transition) then we can estimate the Lindemann number required for a DW factor to reduce the scattering from the vortex liquid to the point where we could no longer observe it. We find $c_{L}$ to be at least $\sim 0.25$, which is within the expected range of values [58]. Hence, the absence of a SANS signal from the vortex liquid does not imply the nonexistence of this phase. While we expect a first-order transition to be sharp, we see that these data do not appear to have a sharp fall to zero at the melting transition, although this may be obscured by the initial fall due to the Debye-Waller factor. We note that this spread also reflects the slight spread in $T_{c}$ of our sample.

\section{CONCLUSIONS}

In conclusion, we report in this paper the microscopic investigation of the structure of vortex matter in a high- $T_{c}$ superconductor in a high magnetic field range. We have observed the structural evolution of the VL rhombic phase in $\mathrm{YBCO}_{7}$, showing no field-independent structure and suggesting a field-driven change in the superconducting states of the $\mathrm{CuO}$ chain layers. The evolution of the VL form factor with field away from the low field London + core model indicates 
that the VL will remain measurable by SANS well above our maximum field, allowing for measurements to be extended further when equipment permits. The deviation of both the field and temperature dependence of the VL form factor from the expected behavior in the London model, combined with the unusual variation in VL structure with increasing temperature, suggests that a static Debye-Waller factor is present in the low field and temperature data. This effect is reduced both at high field and temperature, and confirms that estimates of the coherence length obtained from lower field data are too long [19]. Observations of the VL at high fields and high temperatures show the effects of thermal displacements on the VL perfection prior to the VL melting transition, and provide different information from macroscopic measurements. Our measurements are consistent with a first-order lattice-to-liquid transition, with the intensity scattered from the vortex lines falling sharply across the transition to an unmeasurably low value in the vortex liquid state. In summary, our study provides a further perspective on vortex matter under extreme conditions.

\section{ACKNOWLEDGMENTS}

We acknowledge funding from the UK EPSRC, the University of Birmingham, the Swiss NCCR, and its program MaNEP; also support from the ILL, where the measurements were performed, and the assistance of $\mathrm{S}$. Mühlbauer and A. Heinemann at SANS-I of FRM-II with additional measurements not reported here.
[1] E. M. Forgan, D. M. Paul, H. A. Mook, P. A. Timmins, H. Keller, S. Sutton, and J. S. Abell, Nature (London) 343, 735 (1990).

[2] P. L. Gammel, D. J. Bishop, G. J. Dolan, J. R. Kwo, C. A. Murray, L. F. Schneemeyer, and J. V. Waszczak, Phys. Rev. Lett. 59, 2592 (1987).

[3] I. Maggio-Aprile, Ch. Renner, A. Erb, E. Walker, and Ø. Fischer, Phys. Rev. Lett. 75, 2754 (1995).

[4] Ø. Fischer, C. Renner, I. Maggio-Aprile, A. Erb, E. Walker, B. Revaz, and J. Y. Genoud, Physica C 282-287, 315 (1997).

[5] J. E. Sonier, R. F. Kiefl, J. H. Brewer, D. A. Bonn, S. R. Dunsiger, W. N. Hardy, R. Liang, W. A. MacFarlane, T. M. Riseman, D. R. Noakes, and C. E. Stronach, Phys. Rev. B 55, 11789 (1997).

[6] J. E. Sonier, J. H. Brewer, R. F. Kiefl, G. D. Morris, R. I. Miller, D. A. Bonn, J. Chakhalian, R. H. Heffner, W. N. Hardy, and R. Liang, Phys. Rev. Lett. 83, 4156 (1999).

[7] O. M. Auslaender, L. Luan, E. W. J. Straver, J. E. Hoffman, N. C. Koshnick, E. Zeldov, D. A. Bonn, R. Liang, W. N. Hardy, and K. A. Moler, Nat. Phys. 5, 35 (2008).

[8] M. Yethiraj, H. A. Mook, G. D. Wignall, R. Cubitt, E. M. Forgan, D. M. Paul, and T. Armstrong, Phys. Rev. Lett. 70, 857 (1993).

[9] M. Yethiraj, H. A. Mook, G. D. Wignall, R. Cubitt, E. M. Forgan, S. L. Lee, D. M. Paul, and T. Armstrong, Phys. Rev. Lett. 71, 3019 (1993).

[10] B. Keimer, F. Doğan, I. A. Aksay, R. W. Erwin, J. W. Lynn, and M. Sarikaya, Science 262, 83 (1993).

[11] B. Keimer, W. Y. Shih, R. W. Erwin, J. W. Lynn, F. Dogan, and I. A. Aksay, Phys. Rev. Lett. 73, 3459 (1994).

[12] E. M. Forgan and S. L. Lee, Phys. Rev. Lett. 75, 1422 (1995).

[13] C. M. Aegerter, S. T. Johnson, W. J. Nuttall, S. H. Lloyd, M. T. Wylie, M. P. Nutley, E. M. Forgan, R. Cubitt, S. L. Lee, D. M. Paul, M. Yethiraj, and H. A. Mook, Phys. Rev. B 57, 14511 (1998).

[14] S.T. Johnson, E.M. Forgan, S.H. Lloyd, C.M. Aegerter, S.L. Lee, R. Cubitt, P.G. Kealey, C. Ager, S. Tajima, A. Rykov, and D. McK. Paul, Phys. Rev. Lett. 82, 2792 (1999).

[15] S. P. Brown, D. Charalambous, E. C. Jones, E. M. Forgan, P. G. Kealey, A. Erb, and J. Kohlbrecher, Phys. Rev. Lett. 92, 067004 (2004).

[16] C. Simon, A. Pautrat, G. Poullain, C. Goupil, C. LeblondHarnois, X. Chaud, and A. Brûlet, Phys. Rev. B 70, 024502 (2004).
[17] J. S. White, S. P. Brown, E. M. Forgan, M. Laver, C. J. Bowell, R. J. Lycett, D. Charalambous, V. Hinkov, A. Erb, and J. Kohlbrecher, Phys. Rev. B 78, 174513 (2008).

[18] J. S. White, V. Hinkov, R. W. Heslop, R. J. Lycett, E. M. Forgan, C. Bowell, S. Strässle, A. B. Abrahamsen, M. Laver, C. D. Dewhurst, J. Kohlbrecher, J. L. Gavilano, J. Mesot, B. Keimer, and A. Erb, Phys. Rev. Lett. 102, 097001 (2009).

[19] J. S. White, R. W. Heslop, A. T. Holmes, E. M. Forgan, V. Hinkov, N. Egetenmeyer, J. L. Gavilano, M. Laver, C. D. Dewhurst, R. Cubitt, and A. Erb, Phys. Rev. B 84, 104519 (2011).

[20] D. N. Basov and T. Timusk, Rev. Mod. Phys. 77, 721 (2005).

[21] D. N. Basov, R. Liang, D. A. Bonn, W. N. Hardy, B. Dabrowski, M. Quijada, D. B. Tanner, J. P. Rice, D. M. Ginsberg, and T. Timusk, Phys. Rev. Lett. 74, 598 (1995).

[22] R. Gagnon, S. Pu, B. Ellman, and L. Taillefer, Phys. Rev. Lett. 78, 1976 (1997).

[23] C. Ager, F. Y. Ogrin, S. L. Lee, C. M. Aegerter, S. Romer, H. Keller, I. M. Savić, S. H. Lloyd, S. J. Johnson, E. M. Forgan, T. Riseman, P. G. Kealey, S. Tajima, and A. Rykov, Phys. Rev. B 62, 3528 (2000).

[24] D. H. Lu, D. L. Feng, N. P. Armitage, K. M. Shen, A. Damascelli, C. Kim, F. Ronning, Z.-X. Shen, D. A. Bonn, R. Liang, W. N. Hardy, A. I. Rykov, and S. Tajima, Phys. Rev. Lett. 86, 4370 (2001).

[25] W. A. Atkinson and J. P. Carbotte, Phys. Rev. B 52, 10601 (1995).

[26] W. A. Atkinson and J. E. Sonier, Phys. Rev. B 77, 024514 (2008).

[27] T. Kondo, R. Khasanov, J. Karpinski, S. M. Kazakov, N. D. Zhigadlo, Z. Bukowski, M. Shi, A. Bendounan, Y. Sassa, J. Chang, S. Pailhés, J. Mesot, J. Schmalian, H. Keller, and A. Kaminski, Phys. Rev. Lett. 105, 267003 (2010).

[28] T. Xiang and J. M. Wheatley, Phys. Rev. Lett. 76, 134 (1996).

[29] K. Zhang, D. A. Bonn, S. Kamal, R. Liang, D. J. Baar, W. N. Hardy, D. Basov, and T. Timusk, Phys. Rev. Lett. 73, 2484 (1994).

[30] J. S. White, C. J. Bowell, A. S. Cameron, R. W. Heslop, J. Mesot, J. L. Gavilano, S. Strässle, L. Mächler, R. Khasanov, C. D. Dewhurst, J. Karpinski, and E. M. Forgan, Phys. Rev. B 89, 024501 (2014).

[31] C. C. Tsuei and J. R. Kirtley, Rev. Mod. Phys. 72, 969 (2000).

[32] J. R. Kirtley et al., Nat. Phys. 2, 190 (2006). 
[33] H. J. H. Smilde, A. A. Golubov, Ariando, G. Rijnders, J. M. Dekkers, S. Harkema, D. H. A. Blank, H. Rogalla, and H. Hilgenkamp, Phys. Rev. Lett. 95, 257001 (2005).

[34] R. Khasanov, S. Strässle, D. Di Castro, T. Masui, S. Miyasaka, S. Tajima, A. Bussmann-Holder, and H. Keller, Phys. Rev. Lett. 99, 237601 (2007).

[35] M. Roulin, A. Junod, A. Erb, and E. Walker, Phys. Rev. Lett. 80, 1722 (1998).

[36] A. Erb, E. Walker, and R. Flükiger, Physica C 258, 9 (1996).

[37] C. T. Lin, W. Zhou, W. Y. Liang, E. Schönherr, and H. Bender, Physica C 195, 291 (1992).

[38] V. Hinkov, P. Bourges, S. Pailhès, Y. Sidis, A. Ivanov, C. D. Frost, T. G. Perring, C. T. Lin, D. P. Chen, and B. Keimer, Nat. Phys. 3, 780 (2007).

[39] A. Erb, A. A. Manuel, M. Dhalle, F. Marti, J. Y. Genoud, B. Revaz, A. Junod, D. Vasumathi, S. Ishibashi, A. Shukla, E. Walker, Ø. Fischer, R. Flükiger, R. Pozzi, M. Mali, and D. Brinkmann, Solid State Commun. 112, 245 (1999).

[40] A. T. Holmes, G. R. Walsh, E. Blackburn, E. M. Forgan, and M. Savey-Bennett, Rev. Sci. Instrum. 83, 023904 (2012).

[41] C. D. Dewhurst, GRASP User Manual, Tech. Rep. No. ILL03DE01T, Institut Laue-Langevin, Grenoble, 2003, available at: www.ill.fr/lss/grasp.

[42] D. K. Christen, F. Tasset, S. Spooner, and H. A. Mook, Phys. Rev. B 15, 4506 (1977).

[43] G. L. Squires, Introduction to the Theory of Thermal Neutron Scattering (Dover, New York, 1996).

[44] V. G. Kogan, M. Bullock, B. Harmon, P. Miranović, Lj. Dobrosavljević-Grujić, P. L. Gammel, and D. J. Bishop, Phys. Rev. B 55, R8693(R) (1997).

[45] V. G. Kogan, P. Miranović, Lj. Dobrosavljević-Grujić, W. E. Pickett, and D. K. Christen, Phys. Rev. Lett. 79, 741 (1997).

[46] M. Yethiraj, D. K. Christen, D. McK. Paul, P. Miranović, and J. R. Thompson, Phys. Rev. Lett. 82, 5112 (1999).

[47] M. Franz, I. Affleck, and M. H. S. Amin, Phys. Rev. Lett. 79, 1555 (1997).
[48] K. M. Suzuki, K. Inoue, P. Miranović, M. Ichioka, and K. Machida, J. Phys. Soc. Jpn. 79, 013702 (2010).

[49] M. Ichioka, A. Hasegawa, and K. Machida, Phys. Rev. B 59, 8902 (1999).

[50] J. Chang, J. S. White, M. Laver, C. J. Bowell, S. P. Brown, A. T. Holmes, L. Maechler, S. Strässle, R. Gilardi, S. Gerber, T. Kurosawa, N. Momono, M. Oda, M. Ido, O. J. Lipscombe, S. M. Hayden, C. D. Dewhurst, R. Vavrin, J. Gavilano, J. Kohlbrecher, E. M. Forgan, and J. Mesot, Phys. Rev. B 85, 134520 (2012).

[51] Y. Wang and H.-H. Wen, Europhys. Lett. 81, 57007 (2008).

[52] R. Cubitt, E. M. Forgan, D. McK Paul, S. L. Lee, J. S. Abell, H. Mook, and P. A. Timmins, Physica B 180-181, 377 (1992).

[53] U. Yaron, P. L. Gammel, D. A. Huse, R. N. Kleiman, C. S. Oglesby, E. Bucher, B. Batlogg, D. J. Bishop, K. Mortensen, K. Clausen, C. A. Bolle, and F. De La Cruz, Phys. Rev. Lett. 73, 2748 (1994).

[54] M. H. S. Amin, M. Franz, and I. Affleck, Phys. Rev. Lett. 84, 5864 (2000).

[55] E. Forgan, M. Wylie, S. Lloyd, S. Lee, and R. Cubitt, Czech. J. Phys. 46, 1571 (1996).

[56] S. Kamal, D. A. Bonn, N. Goldenfeld, P. J. Hirschfeld, R. Liang, and W. N. Hardy, Phys. Rev. Lett. 73, 1845 (1994).

[57] A. Junod, M. Roulin, J.-Y. Genoud, B. Revaz, A. Erb, and E. Walker, Physica C: Superconductivity 275, 245 (1997).

[58] J. Kierfeld and V. Vinokur, Phys. Rev. B 69, 024501 (2004).

[59] T. Nishizaki and N. Kobayashi, Supercond. Sci. Tech. 13, 1 (2000).

[60] K. Shibata, T. Nishizaki, T. Sasaki, and N. Kobayashi, Phys. Rev. B 66, 214518 (2002).

[61] R. Cubitt, E. M. Forgan, G. Yang, S. L. Lee, D. M. Paul, H. A. Mook, M. Yethiraj, P. H. Kes, T. W. Li, A. A. Menovsky, Z. Tarnawski, and K. Mortensen, Nature 365, 407 (1993).

[62] C. J. Bowell, R. J. Lycett, M. Laver, C. D. Dewhurst, R. Cubitt, and E. M. Forgan, Phys. Rev. B 82, 144508 (2010). 